\title{
Microbleeds are associated with depressive symptoms in Alzheimer's disease
}

\author{
Anna E. Leeuwis ${ }^{\mathrm{a}, *}$, Niels D. Prins ${ }^{\mathrm{a}}$, Astrid M. Hooghiemstra ${ }^{\mathrm{a}}$, Marije R. Benedictus ${ }^{\mathrm{a}}$, \\ Philip Scheltens ${ }^{\mathrm{a}}$, Frederik Barkhof ${ }^{\mathrm{b}, \mathrm{c}}$, Wiesje M. van der Flier ${ }^{\mathrm{a}, \mathrm{d}}$ \\ ${ }^{a}$ Alzheimer Center and Department of Neurology, Amsterdam Neuroscience, VU University Medical Center, Amsterdam, The Netherlands \\ ${ }^{b}$ Department of Radiology and Nuclear Medicine, Amsterdam Neuroscience, VU University Medical Center, Amsterdam, The Netherlands \\ ${ }^{c}$ Institutes of Neurology and Healthcare Engineering, University College London, London, United Kingdom \\ ${ }^{d}$ Department of Epidemiology and Biostatistics, VU University Medical Center, Amsterdam, The Netherlands
}

\begin{abstract}
Introduction: Co-occurrence of cerebrovascular disease and depression led to the "vascular depression hypothesis". White matter hyperintensities (WMHs) have been associated with depressive symptoms in population-based studies. We studied the association between small vessel disease and depressive symptoms in a memory clinic population.

Methods: We included $>2000$ patients with subjective cognitive decline (SCD), mild cognitive impairment, and Alzheimer's disease (AD). Magnetic resonance imaging was rated for WMHs, lacunes, and microbleeds. Depressive symptoms were assessed using the Geriatric Depression Scale. We performed logistic regression analysis.

Results: Depressive symptoms were present in AD: 17\%; mild cognitive impairment: 25\%; and SCD: $23 \%$. SCD patients with WMHs showed higher propensity of depressive symptoms than AD patients with WMHs. AD patients with microbleeds were more likely to have depressive symptoms compared with $\mathrm{AD}$ patients without microbleeds (odds ratio $=1.70$; 95\% confidence interval: $1.08-2.68$ ).

Discussion: Microbleeds are associated with depressive symptoms in AD, supporting a potential role of cerebral amyloid angiopathy in the occurrence of depressive symptoms in AD.

(C) 2017 The Authors. Published by Elsevier Inc. on behalf of the Alzheimer's Association. This is an open access article under the CC BY-NC-ND license (http://creativecommons.org/licenses/by-nc-nd/ 4.0/).
\end{abstract}

Keywords: $\quad$ Small vessel disease; White matter hyperintensities; Lacunes; Microbleeds; Depressive symptoms; Alzheimer's disease

\section{Introduction}

Depressive symptoms are common in older people and have been associated with cognitive and functional impairment and lower quality of life $[1,2]$. Depressive symptoms in older people are often referred to as late-life depression and are related to an increased risk of dementia [3,4]. Approximately $30 \%$ of patients with dementia experience depressive symptoms [2]. Cerebrovascular disease often co-

*Corresponding author. Tel.: +31204440816; Fax: +31204448529.

E-mail address: a.leeuwis@vumc.nl exists with Alzheimer's disease (AD). Cerebral small vessel disease (CSVD) is the most common vascular cause of dementia and a major contributor to mixed dementia [5]. Magnetic resonance imaging (MRI) markers of CSVD include white matter hyperintensities (WMHs), lacunes, and microbleeds [6]. Associations between depressive symptoms and CSVD have been found cross-sectionally and longitudinally in (healthy) older people and have led to the "vascular depression hypothesis" in late-life depression [7-14]. Depressive symptoms could be an entity on their own-unrelated to cognitive decline and dementia, but depressive symptoms have also been hypothesized to be a prodromal, early manifestation of neurodegeneration or a risk factor for 
dementia [15]. Alternatively, depressive symptoms could be a psychological reaction to perceived cognitive impairment.

Studies investigating the relationship between depressive symptoms and CSVD are mostly limited to large populationbased studies $[3,7,10,16]$, in patients without cognitive impairment. This study is among the first using a large cohort of memory clinic patients. We hypothesize that the relationship between CSVD and depressive symptoms is modulated by disease severity and expect this relationship most prominently in nondemented subjects. To test this hypothesis, we investigated in a cross-sectional study whether MRI markers of CSVD were associated with depressive symptoms in patients with subjective cognitive decline (SCD), mild cognitive impairment (MCI), and AD.

\section{Methods}

\subsection{Subjects}

We included 2136 patients (810 SCD, $488 \mathrm{MCI}$, and 838 AD patients) with available MRI scans and Geriatric Depression Scale (GDS) scores from the memory clinicbased Amsterdam Dementia Cohort [17].

All patients visited the memory clinic between August 2001 and September 2016 and underwent standardized brain MRI. All patients underwent a 1-day standardized dementia screening that included medical history, physical and neurological examinations, screening laboratory tests, neuropsychological assessment, and standardized brain MRI. Clinical diagnosis was established by consensus in a multidisciplinary team. AD patients met the NINCDS-ADRDA criteria (proposed by the National Institute of Neurological and Communicative Disorders and Stroke and the Alzheimer's Disease and Related Disorders Association) for probable AD [18] and also met the core clinical criteria for probable AD proposed by the National Institute on Aging-Alzheimer's Association workgroup [19]. Diagnosis of MCI was based on the Petersen and National Institute on Aging-Alzheimer's Association criteria for MCI [20,21]. Patients were considered to have SCD when they presented with cognitive complaints, and results of clinical assessments were normal (i.e., criteria for MCI or psychiatric disorder were not fulfilled and other underlying neurologic diseases were ruled out). For all patients, history of depression, the use of antidepressants (e.g., selective serotonin reuptake inhibitors [SSRIs], tricyclic antidepressants, monoamine oxidase inhibitors), and the presence of vascular risk factors (i.e., hypertension, diabetes mellitus, and hypercholesterolemia) were determined based on self-reported medical history and medication use. Smoking status was dichotomized into never and former or current smoker. Level of education was classified according to the system of Verhage ranging from 1 to 7 (low to highly educated) [22]. The study was approved by the medical ethics committee of the VU University Medical Center. All patients provided written informed consent for their data to be used for research purposes.

\subsection{Evaluation of depressive symptoms}

Depressive symptoms were assessed using the 15-item self-reported GDS, which has a maximum score of 15 [23]. The GDS-15 is frequently used in clinical practice and research and is a valid and reliable screening instrument for depressive symptoms in older people [23]. A systematic review found a sensitivity of 0.89 and specificity of 0.77 of the GDS-15 at a cutoff score of 5 [24]. In our study, the GDS was orally administered to patients by a neuropsychologist. We classified patients as having depressive symptoms if their score on the GDS was 5 or higher.

\subsection{Evaluation of MRI markers}

MRI was performed on 1.0T $(n=548), 1.5 \mathrm{~T}(n=189)$, or 3.0T $(n=1391)$ scanners. The MRI protocol included T1weighted, T2-weighted, fluid-attenuated inversion recovery (FLAIR) and gradient echo T2*-weighted images. The severity of WMHs using the Fazekas scale was determined on the FLAIR sequence (possible range: 0-3) [25] and was dichotomized into absent (Fazekas $0-1$ ) or present (Fazekas 2-3). WMH data were available for 2128 patients. Lacunes were defined as sharply demarcated deep lesions with CSF-like signal on all sequences and were dichotomized into absent or present ( $\geq 1$ lacune). Lacune count was available for 2074 patients. Microbleeds are small dot-like hypointense lesions on T2*-weighted MRI [6]. Microbleed count was dichotomized into absent or present ( $\geq 1$ microbleed). Microbleed count was available for 2090 patients.

We classified patients as having CSVD if their MRI showed presence of WMHs (Fazekas score $\geq 2$ ), presence of lacunes $(\geq 1$ lacune), and/or presence of microbleeds ( $\geq 1$ microbleed). Global cortical atrophy (GCA) was defined on axial FLAIR images (range 0-3) and dichotomized into absent (0-1) or present (2-3) [26]. Medial temporal lobe atrophy (MTA) was determined on coronal T1weighted images using the Scheltens scale (range 0-4) [27], the mean of left and right MTA scores was dichotomized into MTA absent $(<1.5)$ or MTA present $(\geq 1.5)$.

\subsection{Evaluation of APOE}

DNA was isolated from 10-mL blood samples in ethylenediaminetetraacetic acid. Apolipoprotein E (APOE) $\varepsilon 4$ genotype was determined with the LightCycler $A P O E$ mutation detection method (Roche Diagnostics $\mathrm{GmbH}$, Mannheim, Germany). APOE was analyzed according to the presence or absence of an APOE \&4 allele. APOE $\varepsilon 4$ data were available for 2020 patients (SCD: 757/810; MCI: 462/488; and AD: 801/838).

\subsection{Statistics}

PASW Statistics 22.0 for Mac (SPSS Inc., Chicago, IL, USA) was used for all statistical analyses. Analyses of 
variance and Pearson $\chi^{2}$ tests were performed to compare groups when appropriate.

We performed logistic regression analysis to investigate associations between markers of CSVD and depressive symptoms using a predefined cutoff of 5 for the GDS score to classify patients as depressed (GDS $\geq 5$ ) or not depressed (GDS $<5$ ). In addition, we performed logistic regression analysis to investigate associations between markers of CSVD and antidepressant use. We adjusted for diagnosis (using dummy variables), age, and sex (model 1). We additionally adjusted for education, MRI field strength, Mini-Mental State Examination score, presence of vascular risk factors (hypertension, hypercholesterolemia, and diabetes mellitus), presence of the APOE $\varepsilon 4$ allele, GCA, and MTA (model 2). In model 3, we additionally adjusted for antidepressant use. To check whether associations with CSVD markers differed according to the diagnostic group, interaction terms (diagnosis $\times$ CSVD marker) were included to model 1 . If these interactions were significant, we show the odds ratio (OR) stratified by diagnostic group. If not significant, the interaction term was removed from the model, and associations across groups are shown. The significance level for the analyses of the outcome variables was set at $<.05$.

\section{Results}

Demographic data and MRI measures are summarized by the diagnostic group in Tables 1 and 2. SCD patients were younger than $\mathrm{MCI}$ and $\mathrm{AD}$ patients. $\mathrm{AD}$ patients were more often female than patients with MCI and SCD. We observed depressive symptoms (i.e., GDS $\geq 5$ ) in $17 \%$ of $\mathrm{AD}$ patients, $25 \%$ of MCI patients, and in $23 \%$ of SCD patients. $\mathrm{AD}$ patients less often used antidepressant medication, compared with MCI patients (AD: $8 \%$ vs. MCI: $11 \% ; P<.05)$. CSVD was present in $40 \%$ of AD patients, $46 \%$ of MCI patients, and in $28 \%$ of SCD patients. AD patients had lower scores on the GDS than MCI and SCD patients (AD: $2.58 \pm 2.38$ vs. MCI: $3.21 \pm 2.91$ and SCD: $3.09 \pm 2.68, P<.05)$. $\mathrm{AD}$ and MCI patients more often had WMHs, lacunes, and microbleeds than SCD patients.

\subsection{Association between structural CSVD markers and depressive symptoms}

Table 3 shows the associations between markers of CSVD and presence of depressive symptoms, using logistic regression analysis. We found a significant interaction between WMHs and diagnosis as well as between microbleeds and diagnosis, suggesting that the likelihood of having depressive symptoms associated with WMHs and microbleeds is different for SCD, MCI, and AD. After stratification for diagnosis, results showed that, although not significant, SCD patients with WMHs were more likely to have depressive symptoms compared with SCD patients without $\mathrm{WMH}(\mathrm{OR}=1.56 ; 95 \%$ confidence interval $[\mathrm{CI}]$ : $0.91-2.68 ; P=.100)$. In contrast, AD patients with WMHs tended to show less propensity of depressive symptoms compared with $\mathrm{AD}$ patients without WMHs $(\mathrm{OR}=.64$; 95\% CI: 0.39-1.04; $P=.075)$. Second, AD patients with

Table 1

Demographics

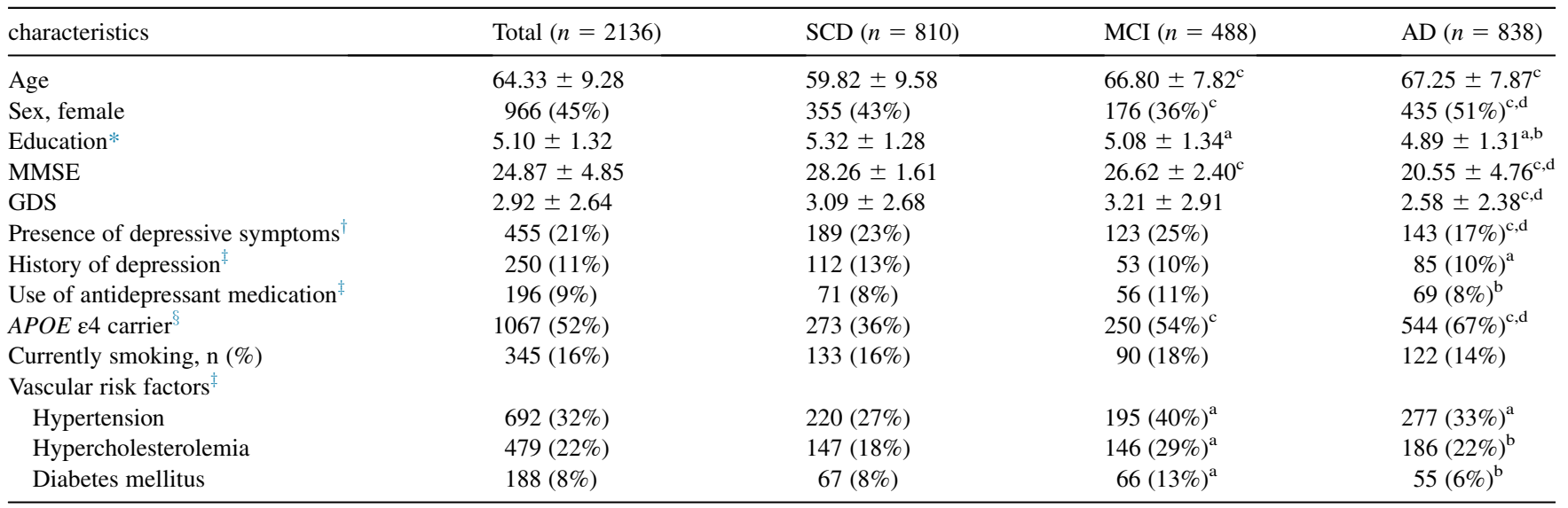

Abbreviations: AD, Alzheimer's disease; ANOVA, analysis of variance; GDS, Geriatric Depression Scale; MCI, mild cognitive impairment; MMSE, MiniMental State Examination; SCD, subjective cognitive decline.

NOTE. One-way ANOVA or $\chi^{2}$ was performed, respectively. Data are presented as mean \pm standard deviation or number (percentage).

NOTE. Significant difference: ${ }^{\mathrm{a}} P<.05$ compared with $\mathrm{SCD} ;{ }^{\mathrm{b}} P<.05$ compared with $\mathrm{MCI}$; ${ }^{\mathrm{c}} P<.001$ compared with SCD; and ${ }^{\mathrm{d}} P<.001$ compared with MCI.

*Level of education was classified according to the system of Verhage ranging from 1 to 7 (low to highly educated).

†Presence of depressive symptoms indicates a score of $\geq 5$ on the GDS.

${ }^{\ddagger}$ History of depression, antidepressant use, and presence of vascular risk factors (i.e., hypertension, hypercholesterolemia, and diabetes mellitus) were determined based on self-reported medical history and medication use.

${ }^{\S}$ APOE \&4 data were available for 2020 patients. 
Table 2

Structural MRI measures

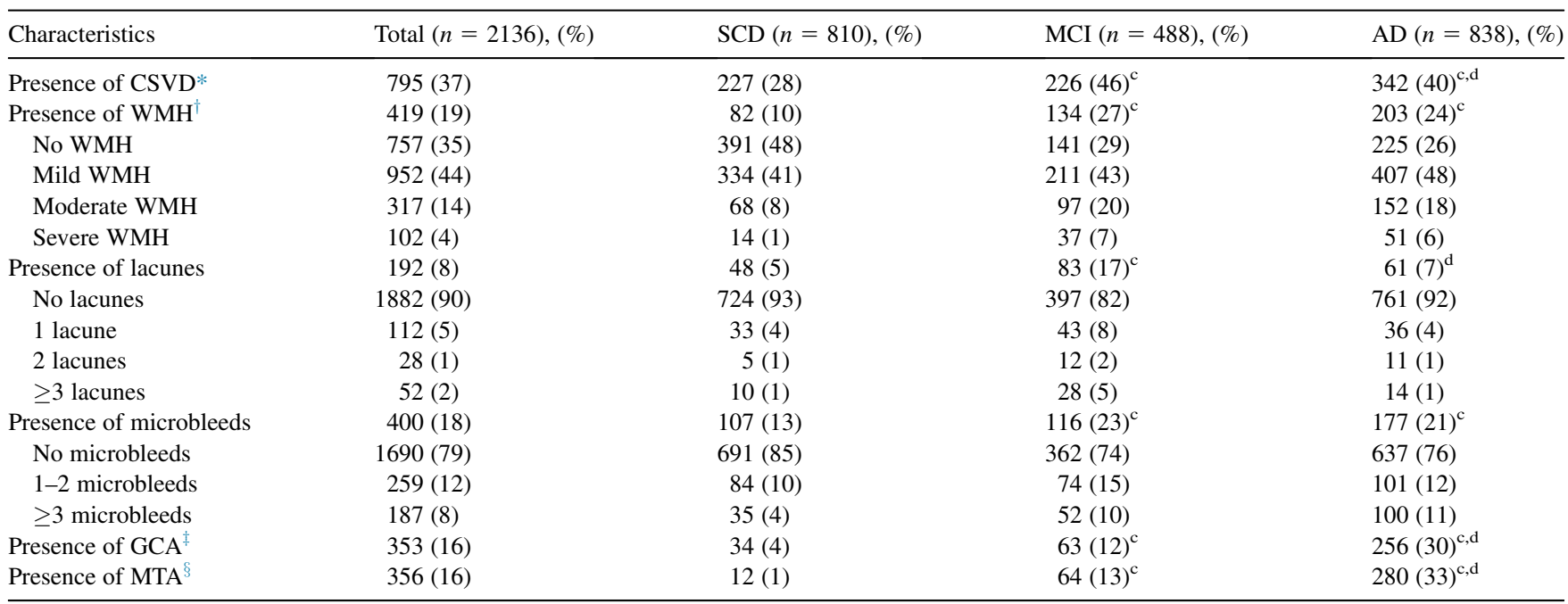

Abbreviations: AD, Alzheimer's disease; ANOVA, analysis of variance; CSVD, cerebral small vessel disease; GCA, global cortical atrophy; MCI, mild cognitive impairment; MTA, medial temporal lobe atrophy; MRI, magnetic resonance imaging; SCD, subjective cognitive decline; WMH, white matter hyperintensity.

NOTE. One-way ANOVA or $\chi^{2}$ were performed, respectively. Data are presented as number (percentage). Significant difference: ${ }^{\mathrm{a}} P<.05$ compared with $\mathrm{SCD} ;{ }^{\mathrm{b}} P<.05$ compared with $\mathrm{MCI} ;{ }^{\mathrm{c}} P<.001$ compared with SCD; and ${ }^{\mathrm{d}} P<.001$ compared with MCI.

*CSVD indicates the presence of WMH and/or the presence of lacunes and/or the presence of microbleeds.

${ }^{\dagger}$ WMHs were rated with the Fazekas scale (0-3) and were dichotomized into absent (Fazekas 0-1) or present (Fazekas 2-3).

${ }^{\ddagger}$ Global cortical atrophy was rated with a visual rating scale (0-3) and was dichotomized into absent (GCA 0-1) or present (GCA 2-3).

${ }^{\S}$ Medial temporal lobe atrophy was rated with a visual rating scale (0-4), and the mean of left and right MTA scores was dichotomized into MTA absent $(<1.5)$ or MTA present $(\geq 1.5)$.

microbleeds were more likely to have depressive symptoms compared with $\mathrm{AD}$ patients without microbleeds $(\mathrm{OR}=1.70 ; 95 \%$ CI: 1.11-2.60; $P<.05)$, whereas such an effect was not present in MCI or SCD. Lacunes were not associated with the presence of depressive symptoms in any of the groups.

When we repeated the analyses with additional adjustment for education, MRI field strength, Mini-Mental State Examination score, presence of vascular risk factors, presence of $A P O E \varepsilon 4$ allele and atrophy (model 2), and with additional adjustment for antidepressant use (model 3), we found similar results. Finally, we performed an additional analysis between the presence of CSVD and depressive symptoms. We found no associations between the presence of CSVD and depressive symptoms (model 1: OR $=1.10$; 95\% CI: $0.87-1.39$ and model $2:$ OR $=1.07 ; 95 \%$ CI: $0.83-1.37$; both $P>.05$ ) (data not shown).

Finally, we did not find an association between antidepressant use and microbleeds in any of the groups (AD: $\mathrm{OR}=0.75$; 95\% CI: $0.38-1.46$; MCI: $\mathrm{OR}=0.63$; $95 \%$ CI: 0.28-1.412; and SCD: OR $=0.92$; 95\% CI: .43-1.98).

\section{Discussion}

The main finding of this study is that microbleeds are related to the prevalence of depressive symptoms in $\mathrm{AD}$ patients, but not in patients with MCI or SCD. Furthermore, the propensity for depressive symptoms is higher in SCD patients with WMHs than in AD patients with WMHs. We found no associations between lacunes and the prevalence of depressive symptoms in any group (Figs. 1 and 2).

Previous studies on depressive symptoms in older people have primarily focused on the relation of WMHs with depression. Population-based studies have firmly established both cross-sectional and longitudinal associations between depressive symptoms and depression and WMH severity. Persons with depression more frequently have WMHs than controls without depression $[8,28]$. Persons with late-life depression have a four-fold higher prevalence of WMHs than persons with early-onset depression or controls [28]. Greater longitudinal increases in WMH volume are associated with poorer outcomes in late-life depression [29]. Presence of WMHs can increase the risk of depression later in life, which supports the "vascular depression hypothesis". Also, previous literature showed that lacunar infarcts have been associated with depressive disorders in healthy older people [30]. The Leukoaraoisis and Disability (LADIS) Study found an association between lacunes in mainly the basal ganglia region and depressive symptoms in older people [9]. An autopsy study in older people with late-life depression, however, found that depression was not related to WMHs or lacunes [31]. In a population-based study in which depression was diagnosed by a structured diagnostic interview, depression was associated with the presence of Lewy bodies, but not with the presence of WMHs and/or lacunes or Alzheimer pathology [32]. These 
Table 3

Logistic regression models for the association between SVD markers and depressive symptoms

\begin{tabular}{|c|c|c|c|c|}
\hline Cerebral SVD marker & All & SCD & MCI & $\mathrm{AD}$ \\
\hline Model 1 & $0.95(0.71-1.26)^{*}$ & $1.56(0.91-2.68)$ & $1.00(0.62-1.62)$ & $0.64(0.39-1.04)$ \\
\hline Model 3 & $0.89(0.65-1.22)^{*}$ & $1.59(0.86-2.92)$ & $0.85(0.49-1.48)$ & $0.58(0.33-1.01)$ \\
\hline \multicolumn{5}{|l|}{ Lacunes } \\
\hline Model 1 & $1.23(0.85-1.78)$ & $1.29(0.64-2.57)$ & $1.26(0.73-2.17)$ & $1.15(0.56-2.38)$ \\
\hline \multicolumn{5}{|l|}{ Microbleeds } \\
\hline Model 1 & $1.20(0.92-1.58)^{*}$ & $0.94(0.56-1.56)$ & $0.98(0.60-1.61)$ & $1.70(1.11-2.60)^{\dagger}$ \\
\hline Model 2 & $1.24(0.93-1.65)$ & $0.95(0.55-1.64)$ & $1.07(0.63-1.81)$ & $1.70(1.08-2.68)^{\dagger}$ \\
\hline Model 3 & $1.29(0.97-1.72)$ & $0.96(0.56-1.66)$ & $1.15(0.67-1.97)$ & $1.79(1.13-2.82)^{\prime}$ \\
\hline
\end{tabular}

Abbreviations: AD, Alzheimer's disease; GCA, global cortical atrophy; MCI, mild cognitive impairment; MMSE, Mini-Mental State Examination; MRI, magnetic resonance imaging; MTA, medial temporal lobe atrophy; SCD, subjective cognitive decline; SVD, small vessel disease; WMH, white matter hyperintensity.

NOTE. Logistic regression analyses with data represented as odds ratios (95\% confidence intervals).

NOTE. Model 1: Adjusted for diagnosis, age, and sex. Model 2: Additionally for education, MRI field strength, MMSE score, presence of vascular risk factors, presence of APOE \&4 allele, GCA, and MTA. Model 3: Additionally for antidepressant use. To check if associations with SVD markers differed according to the diagnostic group, interaction terms (dummy diagnosis*SVD marker) were included in the model. If these interactions were significant, we showed the odds ratio stratified by the diagnostic group for all models. If not significant, the interaction term was removed from the model, and associations across groups are shown. The significance level for the analyses of the outcome variables is set at $<.05$.

*Significant interaction term; subsequently stratification for diagnosis.

${ }^{\dagger} P<.05$.

neuropathological studies show that late-life depression can develop in the absence of vascular abnormalities. In our study, we expected to find associations between depressive symptoms and WMHs. By contrast, we did not find a significant association between depressive symptoms and WMHs in any group. For AD patients, we even found an OR in a direction opposite to what we expected, namely that $\mathrm{AD}$ patients with WMHs had a decreased risk of depressive symptoms compared with AD patients without WMHs. An explanation for these results could be the use of the Fazekas

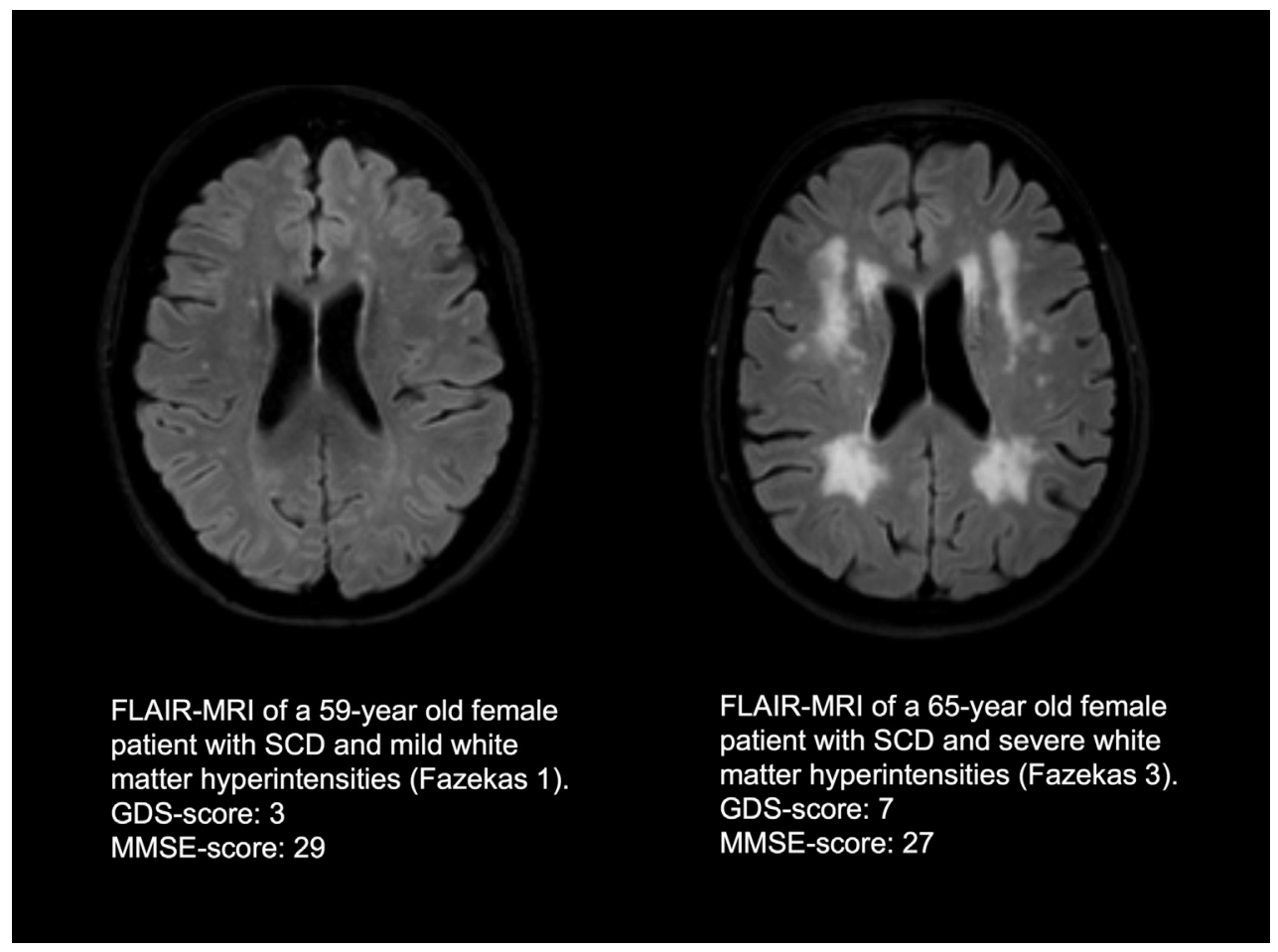

Fig. 1. Examples of FLAIR-MRI scans. Abbreviations: FLAIR, fluid-attenuated inversion recovery; GDS, Geriatric Depression Scale; MMSE, Mini-Mental State Examination; MRI, magnetic resonance imaging; SCD, subjective cognitive decline. 


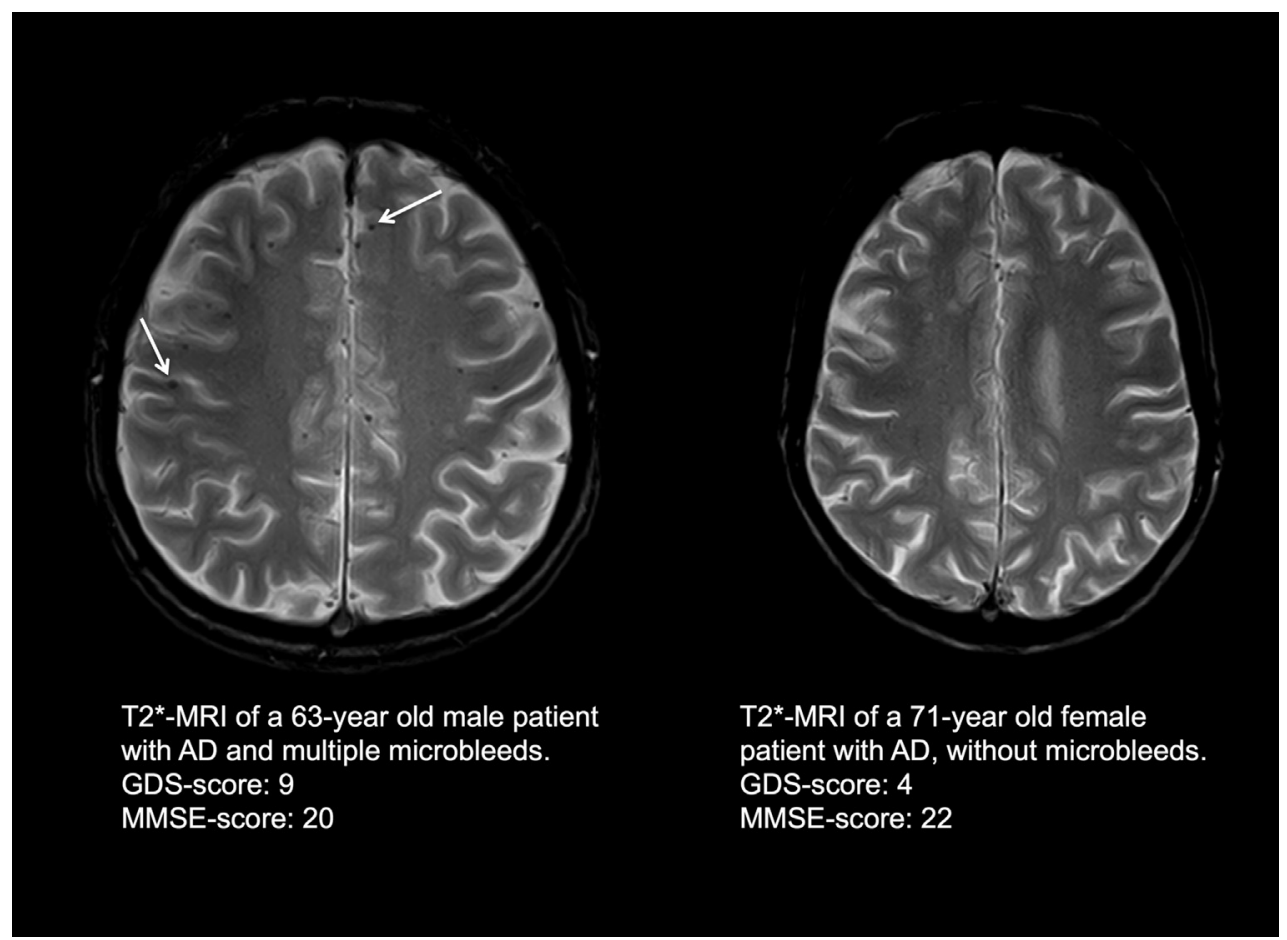

Fig. 2. Examples of T2*-MRI scans (arrows indicate microbleed). Abbreviations: AD, Alzheimer's disease; GDS, Geriatric Depression Scale; MMSE, MiniMental State Examination; MRI, Magnetic resonance imaging.

scale, a widely used visual rating scale for WMHs. Although the Fazekas scales correlates well with volumetry assessment [33], visual rating scales such as the Fazekas scale show ceiling effects and nonlinearity of the data. A second explanation could be the use of the GDS-15 as measure of depressive symptoms in our study. Cognitive impairments in MCI and AD patients may affect the sensitivity and specificity and hence influence the diagnostic accuracy of GDS15 [34]. However, the design of the GDS-15 with the yes/no format and the oral administration by a neuropsychologist makes it easy to use, even for patients with cognitive impairment. Furthermore, the course of depressive symptoms is heterogeneous across older people, and therefore a single assessment of depressive symptoms could have led to overestimate or underestimate of the presence of depression. We did not evaluate the presence of a depressive disorder by a clinical psychiatric interview. Other studies use different questionnaires or a (semi-)structured interview to define the presence for depressive symptoms, for example, the Center for Epidemiological Studies Depression Scale, the Hamilton Rating Scale for Depression or the MontgomeryAsberg Depression Rating Scale. In our study, we used a cutoff for the GDS-15 to classify patients with a score of 5 or higher as having depressive symptoms [24]. It is possible that the cutoff in our study was too low, which potentially overestimated the number of patients in our cohort as having depressive symptoms. However, additional linear regression analyses with a continuously used GDS showed the same results (data shown in Supplementary Table 1). Finally, the use of different scanners with variability in MRI field strength could have induced variability in the results. By correcting for MRI field strength in our analyses, we tried to minimize the potential effect of variability in MRI field strength.

We found a relationship between depressive symptoms and microbleeds in AD. Earlier studies regarding the relationship between depressive symptoms and microbleeds found mixed results. A population-based study with a cross-sectional design found an association between deep microbleeds and depression [30], whereas a longitudinal population-based study in healthy older people found no such association [35]. In patients with stroke, lobar microbleeds have been associated with post-stroke depression [36]. The Rotterdam Study reported that cross-sectionally, antidepressant use was not associated with prevalence of microbleeds, but use of SSRIs predisposed for an increased risk of novel (incident) microbleeds [37,38]. In our cohort, like in the Rotterdam Study, we found no association between prevalence of microbleeds and antidepressant use. It is conceivable that antidepressant use, particularly SSRIs, would further increase the risk of incident microbleeds, but our current results do not allow to make such inferences.

Microbleeds are focal deposits of hemosiderin that indicate previous microhemorrhages. Microbleeds may occur in deep or lobar locations, depending on their pathophysiology. Cardiovascular risk factors (i.e., hypertension) and the presence of cerebral SVD markers (lacunes and WMHs) have been associated with central or infratentorial microbleeds [39]. Lobar microbleeds have been associated 
with cerebral amyloid angiopathy (CAA), a major cause of cognitive impairments in older people [40]. The presence of multiple lobar microbleeds has been shown to be highly specific for CAA in older people. Hereditary cerebral hemorrhage with amyloidosis-Dutch type (HCHWA-D) is an autosomal dominant disease with a similar underlying pathology of amyloid $\beta$ deposition to that in sporadic CAA. Symptomatic HCHWA-D subjects show higher scores on anxiety and depression scales than presymptomatic HCHWA-D subjects and controls [41]. Our results suggest that the underlying mechanism for the relation between depressive symptoms and microbleeds might potentially be the presence of CAA and imply that the predisposition of vascular depression is not only driven by a vasculoischemic pathway but also by a CAA pathway.

Owing to the absence of information about the specific lesion location of WMHs in our analyses, we could not differentiate between periventricular and deep WMHs [6]. Likewise, we did not have information about the localization of microbleeds. The differences in observed associations between CSVD markers and depression in population-based studies and our study could potentially be due to differences in anatomical locations, pathogenesis, or risk factors for the CSVD markers.

In conclusion, our study investigated the association between depressive symptoms and MRI markers of CSVD in a memory clinic cohort. We did not find evidence for the relation between WMHs and depressive symptoms in $\mathrm{AD}$, suggesting that the "vascular depression hypothesis" is limited to cognitively normal elderly. The observed relation between microbleeds and depressive symptoms in $\mathrm{AD}$ suggests that CAA could be an underlying mechanism in the etiology of depressive symptoms in AD.

\section{Acknowledgments}

Research of the VUmc Alzheimer Center is part of the neurodegeneration research program of Amsterdam Neuroscience. The VUmc Alzheimer Center is supported by Alzheimer Nederland and Stichting VUmc Fonds. The clinical database structure was developed with funding from Stichting Dioraphte. We acknowledge the support from The Netherlands CardioVascular Research Initiative: the Dutch Heart Foundation (CVON 2012-06 Heart Brain Connection), Dutch Federation of University Medical Centres, The Netherlands Organisation for Health Research and Development, and the Royal Netherlands Academy of Sciences.

A.E.L., A.M.H., and M.R.B. report no disclosures. N.D.P. serves on the advisory board of Boehringer Ingelheim and Probiodrug and on the DSMB of AbbVie's M15-566 trial. He has provided consultancy services for Sanofi, Takeda, and Kyowa Kirin Pharmaceutical Development. N.D.P. receives research support from Alzheimer Nederland (project number WE.03-2012-02). N.D.P. is the CEO and co-owner of the Alzheimer Research Center, Amsterdam, The
Netherlands. P.S. has acquired grant support (for the institution) from GE Healthcare and Piramal. In the past 2 years, he has received consultancy/speaker fees (paid to the institution) from Novartis, Probiodrug, Biogen, Roche, and EIP Pharma, LLC. F.B. serves as a consultant for Biogen-Idec, Janssen Alzheimer Immunotherapy, Bayer-Schering, Merck Serono, Roche, Novartis, Genzume, and Sanofi-Aventis. F.B. has received sponsoring from EU-H2020, NWO, SMSR, TEVA, Novartis, Toshiba, and IMI. He serves on the editorial boards of Radiology, Brain, Neuroradiology, MSJ, and Neurology. W.M.v.d.F. performs contract research for Boehringer Ingelheim and has been an invited speaker at Boehringer Ingelheim. Research programs of W.M.v.d.F. have been funded by ZonMW, NWO, EU-FP7, Alzheimer Nederland, Cardiovasculair Onderzoek Nederland, Stichting Dioraphte, Gieskes-Strijbis Fonds, Boehringer Ingelheim, Piramal Neuroimaging, Roche BV, Janssen Stellar, and Combinostics. All funding is paid to her institution.

Sources of funding: A.E.L. and A.M.H. are appointed on a grant from The Netherlands CardioVascular Research Initiative: the Dutch Heart Foundation (CVON 2012-06 Heart Brain Connection).

\section{Supplementary data}

Supplementary data related to this article can be found at https://doi.org/10.1016/j.dadm.2017.11.006.

\section{RESEARCH IN CONTEXT}

1. Systematic review: We reviewed the literature using PubMed for articles regarding depressive symptoms and small vessel disease in older people. We cite several studies showing a link between white matter hyperintensities and depressive symptoms in healthy elderly. In our study, we investigated the association between small vessel disease markers and depressive symptoms in a memory clinic cohort.

2. Interpretation: We found associations between microbleeds and depressive symptoms in Alzheimer's disease (AD), but not such an effect in nondemented patients. This could suggest a potential role of cerebral amyloid angiopathy in the etiology of depressive symptoms in $\mathrm{AD}$.

3. Future directions: Further studies should investigate the potential role of cerebral amyloid angiopathy in depression in AD. In addition, we are currently investigating the association between white matter hyperintensities location, using lesion symptom mapping, and depression in AD. 


\section{References}

[1] Sivertsen H, Bjørkløf GH, Engedal K, Selbæk G, Helvik AS. Depression and quality of life in older persons: a review. Dement Geriatr Cogn Disord 2015;40:311-39.

[2] Blazer DG. Depression in late life: review and commentary. J Gerontol 2003;58:249-65.

[3] Diniz BS, Butters MA, Albert SM, Dew MA, Reynolds CF. Late-life depression and risk of vascular dementia and Alzheimer's disease: systematic review and meta-analysis of community-based cohort studies. Br J Psychiatry 2013;202:329-35.

[4] Wilson RS, Barnes LL, Schneider JA, Bienias JL, Evans DA, Bennett DA. Depressive symptoms, cognitive decline, and risk of AD in older persons. Neurology 2003;60:1777-81.

[5] Pantoni L. Cerebral small vessel disease: from pathogenesis and clinical characteristics to therapeutic challenges. Lancet Neurol 2010; 9:689-701.

[6] Wardlaw JM, Smith EE, Biessels GJ, Cordonnier C, Fazekas F, Frayne R, et al. Neuroimaging standards for research into small vessel disease and its contribution to ageing and neurodegeneration. Lancet Neurol 2013;12:822-38.

[7] Ikram MA, Luijendijk HJ, Vernooij MW, Hofman A, Niessen WJ. Vascular brain disease and depression in the elderly. Epidemiology 2010;21:1999-2000.

[8] Firbank MJ, Teodorczuk A, van der Flier WM, Gouw AA, Wallin A, Erkinjuntti T, et al. Relationship between progression of brain white matter changes and late-life depression: 3-year results from the LADIS Study. Br J Psychiatry 2012;201:40-5.

[9] O’Brien JT, Firbank MJ, Krishnan MS, van Straaten ECW, van der Flier WM, Petrovic K, et al. White matter hyperintensities rather than lacunar infarcts are associated with depressive symptoms in older people: the LADIS Study. Am J Geriatr Psychiatry 2006;14:834-41.

[10] De Groot JC, de Leeuw F-E, Oudkerk M, Hofman A, Jolles J, Breteler MMB. Cerebral white matter lesions and depressive symptoms in elderly adults. Arch Gen Psychiatry 2000;57:1071.

[11] Thomas AJ, O'Brien JT, Davis S, Ballard C, Barber R, Kalaria RN, et al. Ischemic basis for deep white matter hyperintensities in major depression: a neuropathological study. Arch Gen Psychiatry 2002; 59:785-92.

[12] Alexopoulos GS, Meyers BS, Young RC, Campbell S, Silbersweig D, Charlson M. "Vascular depression" hypothesis. Arch Gen Psychiatry 1997;54:915-22.

[13] Baldwin RC. Is vascular depression a distinct sub-type of depressive disorder? A review of causal evidence. Int J Geriatr Psychiatry 2005;20:1-11.

[14] Krishnan KRR, Taylor WD, McQuoid DR, MacFall JR, Payne ME, Provenzale JM, et al. Clinical characteristics of magnetic resonance imaging-defined subcortical ischemic depression. Biol Psychiatry 2004;55:390-7.

[15] Chen P, Ganguli M, Mulsant BH, DeKosky ST. The temporal relationship between depressive symptoms and dementia. Arch Gen Psychiatry 1999;56:261.

[16] Barnes DE, Alexopoulos GS, Lopez OL, Williamson JD, Yaffe K. Depressive symptoms, vascular disease, and mild cognitive impairment. Arch Gen Psychiatry 2006;63:273-80.

[17] Van der Flier WM, Pijnenburg YA, Prins N, Lemstra AW, Bouwman FH, Teunissen CE, et al. Optimizing patient care and research: the Amsterdam Dementia Cohort. J Alzheimers Dis 2014;41:313-27.

[18] McKhann G, Drachman D, Folstein M, Katzman R. Clinical diagnosis of Alzheimer's disease: report of the NINCDS-ADRDA Work Group under de auspices of Department of Health and Human Services Task Force on Alzheimer's disease. Neurology 1984; 34:939-44.
[19] McKhann GM, Knopman DS, Chertkow H, Hyman BT, Jack CR, Kawas CH, et al. The diagnosis of dementia due to Alzheimer's disease: recommendations from the National Institute on Aging-Alzheimer's Association workgroups on diagnostic guidelines for Alzheimer's disease. Alzheimers Dement 2011;7:263-9.

[20] Petersen RC, Stevens JC, Ganguli M, Tangalos EG, Cummings JL, Dekosky ST, et al. Practice parameter: early detection of dementia: Mild Cognitive Impairment (an evidence-based review). Neurology 2001;56:1133-42.

[21] Albert MS, DeKosky ST, Dickson D, Dubois B, Feldman HH, Fox NC, et al. The diagnosis of mild cognitive impairment due to Alzheimer's disease: recommendations from the National Institute on Aging-Alzheimer's Association workgroups on diagnostic guidelines for Alzheimer's disease. Alzheimers Dement 2011;7:270-9.

[22] Verhage F. Intelligentie en leeftijd: Onderzoek bij Nederlanders van 12-77 jaar [in Dutch]. Van Gorcum: Assen, the Netherlands; 1964.

[23] Yesavage JA, Sheikh JI. Geriatric Depression Scale (GDS): recent evidence and development of a shorter version. Clin Gerontol 1986; 5:165-73.

[24] Pocklington C, Gilbody S, Manea L, Mcmillan D. The diagnostic accuracy of brief versions of the Geriatric Depression Scale: a systematic review and meta-analysis. Int J Geriatr Psychiatry 2016; 31:837-57.

[25] Fazekas F, Chawluk JB, Hurtig HI, Zimmerman RA. MR signal abnormalities at $1.5 \mathrm{~T}$ in Alzheimer's dementia and normal aging. Am J Neuroradiol 1987;8:421-6.

[26] Pasquier F, Leys D, Weerts JGE, Mounier-Vehier F, Barkhof F, Scheltens P. Inter- and intraobserver reproducibility of cerebral atrophy assessment on MRI scans with hemispheric infarcts. Eur J Neurol 1996;36:268-72.

[27] Scheltens P, Launer LJ, Barkhof F, Weinstein HC, van Gool WA. Visual assessment of medial temporal lobe atrophy on magnetic resonance imaging: interobserver reliability. J Neurol 1995;242:557-60.

[28] Herrmann LL, Herrmann LL, Le Masurier M, Le Masurier M, Ebmeier KP, Ebmeier KP, et al. White matter hyperintensities in late life depression: a systematic review. J Neurol Neurosurg Psychiatry 2008;79:619-24.

[29] Taylor WD, Steffens DC, MacFall JR, McQuoid DR, Payne ME, Provenzale JM, et al. White matter hyperintensity progression and latelife depression outcomes. Arch Gen Psychiatry 2003;60:1090-6.

[30] Direk N, Perez HS, Akoudad S, Verhaaren BFJ, Niessen WJ, Hofman A, et al. Markers of cerebral small vessel disease and severity of depression in the general population. Psychiatry Res Neuroimaging 2016;253:1-6.

[31] Xekardaki A, Santos M, Hof P, Kövari E, Bouras C, Giannakopoulos P. Neuropathological substrates and structural changes in late-life depression: the impact of vascular burden. Acta Neuropathol 2012; 124:453-64.

[32] Tsopelas C, Stewart R, Savva GM, Brayne C, Ince P, Thomas A, et al. Neuropathological correlates of late-life depression in older people. $\mathrm{Br}$ J Psychiatry 2011;198:109-14.

[33] Kapeller P, Barber R, Vermeulen RJ, Adèr H, Scheltens P, Freidl W, et al. Visual rating of age-related white matter changes on magnetic resonance imaging: scale comparison, interrater agreement, and correlations with quantitative measurements. Stroke 2003;34:441-5.

[34] De Craen AJM, Heeren TJ, Gussekloo J. Accuracy of the 15-item geriatric depression scale (GDS-15) in a community sample of the oldest old. Int J Geriatr Psychiatry 2003;18:63-6.

[35] Van Sloten TT, Sigurdsson S, van Buchem MA, Phillips CL, Jonsson PV, Ding J, et al. Cerebral small vessel disease is associated with a higher incidence of depressive symptoms in a general elderly population: the AGES-Reykjavik Study. Am J Psychiatry 2015; 172:570-8 
[36] Tang WK, Chen YK, Lu JY, Chu WCW, Mok VCT, Ungvari GS, et al. Cerebral microbleeds and depression in lacunar stroke. Stroke 2011; 42:2443-6.

[37] Akoudad S, Aarts N, Noordam R, Ikram MA, Tiemeier H, Hofman A. Antidepressant use is associated with an increased risk of developing microbleeds. Stroke 2016;47:251-4.

[38] Aarts N, Akoudad S, Noordam R, Hofman A, Ikram MA, Stricker $\mathrm{BH}$, et al. Inhibition of serotonin reuptake by antidepressants and cerebral microbleeds in the general population. Stroke 2014;45:1951-7.
[39] Romero JR, Preis SR, Beiser A, Decarli C, Viswanathan A, MartinezRamirez S, et al. Risk factors, stroke prevention treatments, and prevalence of cerebral microbleeds in the Framingham Heart Study. Stroke 2014;45:1492-4.

[40] Viswanathan A, Greenberg SM. Cerebral amyloid angiopathy in the elderly. Ann Neurol 2011;70:871-80.

[41] Van Rooden S, van Opstal AM, Labadie G, Terwindt GM, Wermer MJH, Webb AG, et al. Early magnetic resonance imaging and cognitive markers of hereditary cerebral amyloid angiopathy. Stroke 2016;47:3041-4. 